H. Kaneta

Nagoya Math. J.

Vol. 78 (1980), 113-136

\title{
IRREDUCIBILITY OF SOME UNITARY REPRESENTATIONS OF THE POINCARÉ GROUP WITH RESPECT TO THE POINCARÉ SUBSEMIGROUP, I
}

\author{
HITOSHI KANETA
}

\section{§1. Introduction}

Since E. Wigner set up a framework of the relativistically covariant quantum mechanics, several aspects of unitary representations of the Poincaré group have been investigated (see [8], [16]). In this paper it will be shown that some unitary representations of the Poincaré group are irreducible, even if they are restricted to the Poincaré semigroup (Theorem 1,2 and 3). As a result of the argument we shall also give the irreducible decomposition of induced representations $\underset{S U(1,1) \uparrow S L(2, C)}{\operatorname{Ind} \pi}$ (see $\S 3$, cf. [3]). Here the Poincaré group $P$ means a semi-direct product between $R_{4}$ and $S L(2, C)$ with the multiplication

$$
(x, g)\left(x^{\prime}, g^{\prime}\right)=\left(x+g^{-1^{*}} x^{\prime} g^{-1}, g g^{\prime}\right) \quad \text { for } x, x^{\prime} \in R_{4} \text { and } g, g^{\prime} \in S L(2, C),
$$

where $x=\left(x_{0}, x_{1}, x_{2}, x_{3}\right)$ is identified with the matrix $\left(\begin{array}{cc}x_{0}-x_{3} & x_{2}-i x_{1} \\ x_{2}+i x_{1} & x_{0}+x_{3}\end{array}\right)$ and $g *$ shows the adjoint of the matrix $g$. The Poincare semigroup $P_{+}$ is the subsemigroup $\left\{(x, g) \in P: x_{0}^{2}-x_{1}^{2}-x_{2}^{2}-x_{3}^{2} \geqslant 0, x_{0} \geqslant 0\right\}$.

We have not yet succeeded in proving that any irreducible unitary representations of $P$ are irreducible with respect to $P_{+}$, but in a lower dimensional case we have the following.

THEOREM 1. Every irreducible unitary representation of the 2dimensional space-time Poincaré group $P(2)$ is irreducible too as the representation restricted to its Poincaré subsemigroup. Here $P(2)$ is the semi-direct product between $\boldsymbol{R}_{2}$ and $\left\{\left(\begin{array}{cc}e^{t / 2} & 0 \\ 0 & e^{-t / 2}\end{array}\right): t \in R\right\}$ with the same multiplication as $P$ under the identification $\left(x_{0}, x_{3}\right) \rightarrow\left(\begin{array}{cc}x_{0}-x_{3} & 0 \\ 0 & x_{0}+x_{3}\end{array}\right)$.

Received September 8, 1978. 
The semigroup is just $\left\{(x, g): x_{0}^{2}-x_{3}^{2} \geqslant 0, x_{0} \geqslant 0\right\}$.

\section{§ 2. Main theorems}

Let us define a bilinear form $\langle$,$\rangle between \boldsymbol{R}_{4}$ and $\hat{\boldsymbol{R}}_{4}$ by $\langle x, \hat{x}\rangle=$ $x_{0} \hat{x}_{0}-x_{1} \hat{x}_{1}-x_{2} \hat{x}_{2}-x_{3} \hat{x}_{3}$. By abuse of symbol, $\langle$,$\rangle stands also for the$ similar bilinear form on $\boldsymbol{R}_{4}$ or $\hat{R}_{4}$. Defining the action of $G=S L(2, C)$ on $\hat{R}_{4}$ by $x \cdot g=g^{*} x g$ (recall the identification), we obtain the well known diagram:

\begin{tabular}{|c|c|c|}
\hline$G$-orbits & fixed points & \multicolumn{1}{|c|}{ little groups } \\
\hline$V_{M}^{ \pm}=\left\{\langle\hat{x}, \hat{x}\rangle=M^{2}, \hat{x}_{0} \gtrless 0\right\}$ & $\pm M\left(\begin{array}{ll}1 & 0 \\
0 & 1\end{array}\right)$ & $S U(2)$ \\
\hline$V_{0}^{ \pm}=\left\{\langle\hat{x}, \hat{x}\rangle=0, x_{0} \gtrless 0\right\}$ & $\pm\left(\begin{array}{ll}1 & 0 \\
0 & 0\end{array}\right)$ & $E(2)=\left\{\left(\begin{array}{ll}e^{i \theta} & 0 \\
\zeta & e^{-i \theta}\end{array}\right)\right\}$ \\
\hline$V_{i M}=\left\{\langle\hat{x}, \hat{x}\rangle=-M^{2}\right\}$ & $M\left(\begin{array}{rr}-1 & 0 \\
0 & 1\end{array}\right)$ & $S U(1,1)=\left\{\left(\begin{array}{ll}\frac{\beta}{\beta} & \alpha\end{array}\right):|\alpha|^{2}-|\beta|^{2}=1\right\}$ \\
\hline$V_{0}=\left\{\langle\hat{x}, \hat{x}\rangle=0, \hat{x}_{0}=0\right\}$ & $\left(\begin{array}{ll}0 & 0 \\
0 & 0\end{array}\right)$ & $S L(2, C)$ \\
\hline
\end{tabular}

$M$ : positive number.

Furthermore there exists a well known correspondence between an irreducible unitary representation of $P$ and a triplet $\left(\omega, G_{0}, \pi\right)$, where $\omega$ stands for one of $G$-orbits and $\pi$ denotes an irreducible unitary representation of the little group $G_{0}$. More precisely, denote $\mathfrak{F}_{\pi}$ the representation space of $\pi$ and $\nu_{\omega}$ the $G$-invariant measure on the homogeneous space $\omega$ $=G_{0} \backslash G$ and let $\mathfrak{S}^{\omega, \pi}$ be a Hilbert space consisting of $\mathfrak{F}_{\pi}$-valued measurable functions on $P$ such that

$$
f\left(\left(x, g_{0}\right)\left(x^{\prime}, g^{\prime}\right)\right)=e^{i\langle x, \hat{x}\rangle} \pi\left(g_{0}\right) f\left(x^{\prime}, g^{\prime}\right) \quad \text { for } g_{0} \in G_{0}
$$

where $\hat{x}$ is a fixed point with the little group $G_{0}$,

$$
\int_{\omega}\|f(x, g)\|_{\tilde{F}_{\pi}}^{2} d \nu_{\omega}<\infty
$$

Then the irreducible unitary representation of $P$ corresponding to the triplet $\left(\omega, G_{0}, \pi\right)$ say $U^{\omega, \pi}$ is realized on $\mathfrak{S}^{\omega, \pi}$ by the formula

$$
U^{o, \pi}(x, g) f\left(x^{\prime}, g^{\prime}\right)=f\left(\left(x^{\prime}, g^{\prime}\right)(x, g)\right) \text {. }
$$


Theorem 2. Irreducible unitary representations of the Poincaré group corresponding to one of the orbits $V_{M}^{ \pm}, V_{0}^{ \pm}$and $V_{0}$ are irreducible as the representation of the Poincaré subsemigroup.

Proof. Let $(U, \mathfrak{F})$ be an irreducible unitary representation of $P$. If it is reducible with respect to $P_{+}$, there exists a non-trivial closed subspace $D \subset \mathscr{F}$ such that $U_{t} D \subsetneq D$ for any $t>0$, where $U_{t}$ denotes $U((t, 0,0,0), e)$. Put $D_{+}=D \ominus \bigcap_{t>0} U_{t} D$ and $\mathscr{S}_{+}=\overline{\bigcup_{t} U_{t} D_{+}}$. Then $D_{+}$is an outgoing subspace of $\mathfrak{S}_{+}$in the sense that

(i) $U_{t} D_{+} \subset D_{+} \quad$ for all $t>0$,

(ii) $\cap U_{t} D_{+}=0$,

(iii) $\frac{t}{\bigcup_{t} U_{t} D_{+}}=\mathfrak{S}_{+} \neq\{0\}$.

In view of Sinai's theorem (Theorem 3.1 in chap. 2 [11]) the restriction $\left(U_{t}, \mathfrak{S}_{+}\right)$, which is a unitary representation of $R$, is unitarily equivalent to some multiple of the regular representation of $\boldsymbol{R}$. Consequently the representation $\left(U_{t}, \mathfrak{S}\right)$ of $\boldsymbol{R}$ must contain at least one regular representation of $R$. On the other hand, making use of (1) and (3) and putting $g^{\prime}=\left(\begin{array}{ll}\alpha & \beta \\ \gamma & \delta\end{array}\right)$, we can verify easily that

$$
U_{t} f\left(x^{\prime}, g^{\prime}\right)=e^{i t \varepsilon M\left(|\alpha|^{2}+|\beta|^{2}\right) / 2} f\left(x^{\prime}, g^{\prime}\right),
$$

where $\varepsilon$ denotes one of constants $\pm 1, \pm M^{-1}$ and 0 . This implies that the spectrum of the selfadjoint operator $\left.i U_{t}^{\prime}\right|_{t=0}$ has either upper or lower bounds. In particular the representation $U_{t}$ never contains the regular representation.

Q.E.D.

We turn now to the representations corresponding to the orbit $V_{i M}$. Since each of them is specified by an irreducible unitary representation of the little group $G_{0}=S U(1,1)$, we summarize those representations after Vilenkin ( $\$ 2$ in chap. VI [17]). All of them can be obtained from algebraic representations on closed subspaces $D$ of $C^{\infty}$-functions $C^{\infty}(T)$ on the 1-dimensional torus $T$. We denote the inner product by (, ).

Theorem 3. Irreducible unitary representations of the Poincaré group $P$ given by the so-called discrete series representations $\pi^{ \pm}(\ell, 0)$ and $\pi^{ \pm}(\ell, 1 / 2)$ of $G_{0}=S U(1,1)$ and the orbit $V_{i M}$ are also irreducible even if they are restricted to the subsemigroup $P_{+}$.

We shall give the proof of Theorem 3 as well as Theorem 1 in the following $\S 5$. 


\begin{tabular}{|c|c|c|c|}
\hline representations $\pi$ & $\pi\left(g_{0}\right) f\left(e^{i \psi}\right)$ for $g_{0}=\left(\begin{array}{cc}\frac{\alpha}{\beta} & \frac{\beta}{\alpha}\end{array}\right)$ & $D$ & $\begin{array}{l}\text { the values of } \\
\left(e^{i \nu \psi}, e^{i \nu \psi}\right) \text { or }\end{array}$ \\
\hline$\pi_{(\ell, 0)} \quad \ell=-1 / 2+i \rho, \rho \geqslant 0$ & $I_{0}=\left|\beta e^{i \psi}+\bar{\alpha}\right|^{2 \ell} f\left(\frac{\alpha e^{i \psi}+\bar{\beta}}{\beta e^{i \psi}+\bar{\alpha}}\right)$ & $C^{\infty}(T)$ & 1 \\
\hline$\pi_{(\ell, 1 / 2)} \quad \ell=-1 / 2+i \rho, \rho>0$ & $I_{1 / 2}=\left|\beta e^{i \psi}+\bar{\alpha}\right|^{2 \ell-1}\left(\beta e^{i \psi}+\bar{\alpha}\right) f\left(\frac{\alpha e^{i \psi}+\bar{\beta}}{\beta e^{i \psi}+\bar{\alpha}}\right)$ & $C^{\infty}(T)$ & 1 \\
\hline$\pi_{(\ell, 0)} \quad-1<\ell<-1 / 2$ & $I_{0}$ & $C^{\infty}(T)$ & $\frac{\Gamma(\ell-\nu+1)}{\Gamma(-\ell-\nu)}$ \\
\hline$\pi_{(\ell, 0)}^{+} \quad \ell=-1,-2, \cdots$ & $I_{0}$ & $\sum_{\nu \geqslant-\iota} a_{\nu} e^{i \nu \psi}$ & $\frac{\Gamma(\ell+\nu+1)}{\Gamma(-\ell+\nu)}$ \\
\hline$\pi_{(\ell, 1 / 2)}^{+} \quad \ell=-1 / 2,-3 / 2, \cdots$ & $I_{1 / 2}$ & $\sum_{\nu \geq-l+1 / 2} a_{\nu} e^{i \nu \dot{v}}$ & $\frac{\Gamma(\ell+\nu+1 / 2)}{\Gamma(-\ell+\nu-1 / 2)}$ \\
\hline$\pi_{\langle\ell, 0\rangle}^{-} \quad \ell=-1,-2, \cdots$ & $I_{0}$ & $\sum_{\nu \geqslant-\ell} a_{\nu} e^{-i \nu \psi}$ & $\frac{\Gamma(\ell+\nu+1)}{\Gamma(-\ell+\nu)}$ \\
\hline$\pi_{(\ell, 1 / 2)}^{-} \quad \ell=-1 / 2,-3 / 2, \cdots$ & $I_{1 / 2}$ & $\sum_{\nu \geqslant-i-1 / 2} a_{\nu} e^{-i \nu \psi}$ & $\frac{\Gamma(\ell+\nu+3 / 2)}{\Gamma(-\ell+\nu+1 / 2)}$ \\
\hline
\end{tabular}

\section{§ 3. Decomposition of unitary representations of $S L(2, C)$}

We begin with reviewing the irreducible unitary representations of $S L(2, C)$ after Naimark [12]. Throughout this section $G$ stands for $S L(2, C)$. For an integer $m$ denote by $L_{m}^{2}(S U(2))$ a subspace of $L^{2}(S U(2))$ consisting of functions $\varphi$ satisfying

$$
\varphi(\gamma u)=e^{-i m t} \varphi(u) \quad \text { for } \gamma=\left(\begin{array}{cc}
e^{+i t / 2} & 0 \\
0 & e^{-i t / 2}
\end{array}\right) .
$$

The irreducible representations $S_{m, \rho}(m \in Z, \rho \in R)$ has a realization on $L_{m}^{2}(S U(2))$ :

$$
V(g) \varphi(u)=-\frac{\alpha(u g)}{\alpha(u \bar{g})} \varphi(u \bar{g}),
$$

where $\alpha(g)=\left|g_{22}\right|^{i \rho-m-2} g_{22}{ }^{m}$ and $u \bar{g}$ denotes a unitary representative of the coset $K u g$ with $K=\left\{\left(\begin{array}{cc}\lambda^{-1} & \mu \\ 0 & \lambda\end{array}\right): \lambda>0, \mu \in C\right\}$. Meanwhile the irreducible representation $D_{\sigma}(0<\sigma<2)$ has a realization on the Hilbert space $\mathfrak{K}_{\sigma}$ in which a subspace $B_{0}$ of bounded functions belonging to $L_{0}^{2}(S U(2))$ is dense:

$$
V(g) \varphi(u)=-\frac{\alpha(u g)}{\alpha(u \bar{g})} \varphi(u \bar{g}) \quad \text { for } \varphi \in B_{0},
$$

where $\alpha(g)=\left|g_{22}\right|^{-\sigma-2}$. We put 


$$
\begin{array}{rlrl}
\omega_{1}(t) & =\left(\begin{array}{ll}
\cos t / 2 & i \sin t / 2 \\
i \sin t / 2 & \cos t / 2
\end{array}\right) & \omega_{2}(t) & =\left(\begin{array}{ll}
\cos t / 2 & -\sin t / 2 \\
\sin t / 2 & \cos t / 2
\end{array}\right) \\
\omega_{3}(t) & =\left(\begin{array}{cc}
e^{i t / 2} & 0 \\
0 & e^{-i t / 2}
\end{array}\right) & \omega_{4}(t)=\left(\begin{array}{ll}
\operatorname{ch} t / 2 & \operatorname{sh} t / 2 \\
\operatorname{sh} t / 2 & \operatorname{ch} t / 2
\end{array}\right) \\
\omega_{5}(t)=\left(\begin{array}{ll}
\operatorname{ch} t / 2 & i \operatorname{sh} t / 2 \\
-i \operatorname{sh} t / 2 & \operatorname{ch} t / 2
\end{array}\right) & \omega_{6}(t)=\left(\begin{array}{cc}
e^{t / 2} & 0 \\
0 & e^{-t / 2}
\end{array}\right) .
\end{array}
$$

We now introduce linear operators associated with a unitary representation $(T, \mathfrak{S})$ of $G$. Define

$$
\begin{aligned}
\omega_{j} & =\left.\frac{d}{d t}\right|_{t=0} T\left(\omega_{j}(t)\right) \quad \text { for } j=1,2, \cdots, 6, \\
H_{ \pm} & =i \omega_{2} \pm \omega_{1}, \quad H_{3}=i \omega_{3}, \quad F_{ \pm}=i \omega_{5} \pm \omega_{4}, \quad F_{3}=i \omega_{6} \\
\Delta_{o} & =-\left(H_{+} H_{-}+H_{-} H_{+}+2 H_{3}^{2}\right) / 2 \\
\Delta & =\left(F_{+} F_{-}+F_{-} F_{+}+2 F_{3}^{2}\right) / 2+\Delta_{0}-1, \\
\Delta^{\prime} & =\left(H_{+} F_{-}+H_{-} F_{+}+F_{+} H_{-}+F_{-} H_{+}+4 H_{3} F_{3}\right) / 2 .
\end{aligned}
$$

More precisely, since the operator $\Delta_{0}$ (resp. $\Delta$ and $\Delta^{\prime}$ ) is essentially selfadjoint with domain $\left\{\right.$ finite sum of $\int_{S U(2)} \varphi_{i}(u) T(u) f_{i} d u: \varphi_{i} \in C^{\infty}(S U(2))$, $\left.f_{i} \in \mathfrak{S C}_{\mathcal{C}}\right\}$ (resp. $\left\{\right.$ finite sum of $\left.\left.\int_{G} \varphi_{i}(g) T(g) f_{i} d g: \varphi_{i} \in C_{0}^{\infty}(G), f_{i} \in \mathfrak{S}\right\}\right)([14])$, we shall use the same letters for their selfadjoint extensions. We denote the domain of an operator $A$ by $D_{A}$. Then $D_{H \pm}$ (resp. $D_{F \pm}$ ) is the intersection $D_{\omega_{1}} \cap D_{\omega_{2}}$ (resp. $D_{\omega_{4}} \cap D_{\omega_{5}}$ ). Clearly $i \omega_{j}$ is a selfadjoint operator with domain $D \omega_{j}$.

Remark. A homomomorphism $A$ from $G$ onto the proper Lorentz group defined by $\Lambda(g) x=g^{*-1} x g^{-1}$ for $x \in \boldsymbol{R}_{4}$ (recall the identification in $\S 1)$ satisfies

$$
\begin{array}{lll}
\Lambda\left(\omega_{1}(t)\right)=a_{2}(-t), & \Lambda\left(\omega_{2}(t)\right)=a_{1}(t), & \Lambda\left(\omega_{3}(t)\right)=a_{3}(t), \\
\Lambda\left(\omega_{4}(t)\right)=b_{2}(-t), & \Lambda\left(\omega_{5}(t)\right)=b_{1}(t), & \Lambda\left(\omega_{6}(t)\right)=b_{3}(t) .
\end{array}
$$

We refer subgroups $a_{i}(t)$ and $b_{i}(t)$ to [12] where a homomorphism $\tilde{\Lambda}(g) x$ $=g x g^{*}$ is used.

We write down explicitly a canonical basis of the representations $S_{m, \rho}$ and $D_{\sigma}$.

Lemma 1. A canonical basis of the representation $S_{m, p}$ is given by $\left\{\varphi_{p, m, \rho}^{k}: p=-k,-k+1, \cdots, k\right.$ and $\left.k=m / 2, m / 2+1, \cdots\right\}$, where 


$$
\varphi_{p, m, \rho}^{k}(u)=\sqrt{2 k+1}\left(\prod_{\nu=m / 2}^{k} \frac{(2 i \nu+\rho)}{\sqrt{4 \nu^{2}+\rho^{2}}}\right) C_{m / 2, p}^{k}(u)
$$

A canonical basis of the representation $D_{\sigma}$ is given by $\left\{\varphi_{p, \sigma}^{k}: p=-k,-k+1\right.$, $\cdots, k$ and $k=0,1, \cdots\}$, where

$$
\varphi_{p, \sigma}^{k}(u)=\sqrt{2 k+1}\left(\prod_{\nu=1}^{k} \frac{i(2 \nu+\sigma)}{\sqrt{4 \nu^{2}-\sigma^{2}}}\right) \sqrt{\frac{\sigma}{2 \pi}} C_{0, p}^{k}(u) .
$$

The function $C_{\mu, \nu}^{k}$ on $S U(2)$ is defined by

$$
\begin{aligned}
C_{\mu, \nu}^{k}(u)=(-1)^{2 k-\mu-\nu} \sqrt{\frac{(k-\mu) !(k+\mu) !}{(k-\nu) !(k+\nu) !}} \sum_{\alpha}\left(\begin{array}{c}
k-\alpha \\
\alpha
\end{array}\right)\left(\begin{array}{c}
k+\nu \\
k-\mu-\alpha
\end{array}\right) \\
\times u_{11}^{\alpha} u_{12}^{k-\mu-\alpha} u_{21}^{k-\nu-\alpha} u_{22}^{\mu+\nu+\alpha},
\end{aligned}
$$

where $\alpha$ ranges from $\max (0,-\mu-\nu)$ up to $\min (k-\mu, k-\nu)$.

Proof. See $\S 11$ and $\S 12$ of [12]. Since we use the homomophism $A$, the canonical basis above differs a little from the one cited in [12].

It seems convenient to reparametrize these representations of $G$ as follows:

$$
\left(T_{m, \lambda}, \mathfrak{S}_{m, \lambda}\right)= \begin{cases}S_{m, \lambda} & \text { for } m \geqslant 1 \\ S_{0,2 \sqrt{\lambda}} & \text { for } m=0, \lambda \geqslant 0 \\ D_{2 \sqrt{-\lambda}} & \text { for } m=0,-1<\lambda<0 \\ \text { unit representation for } m=0, \lambda=-1 .\end{cases}
$$

Thus the representation $\left(T_{m, \lambda}, \mathscr{S}_{m, \lambda}\right)$ has the canonical basis $f_{\nu, m, \lambda}^{k}$ in accordance with Lemma 1 and it holds that

$$
\Delta=-\left(\frac{m}{2}\right)^{2}+\lambda, \quad \Delta^{\prime}=-\frac{m}{2} \lambda .
$$

Furthermore, putting $\ell_{0}=\{(0, \lambda):-1 \leqslant \lambda\}$ and $\ell_{m}=\{(m, \lambda): \lambda \in \boldsymbol{R}\}$ for positive integer $m$, we can identify the dual space $\hat{G}$ with a Borel subset $\sum_{m \geqq 0} \ell_{m}$ in $\boldsymbol{R}_{2}$ (18.9.13 [4]).

Lemma 2. Denote $\left\{f_{\nu, m, \lambda}^{k}\right\}$ the canonical basis of the representation $\left(T_{m, \lambda}, \mathfrak{S}_{m, \lambda}\right)$ then it holds that
(i ) $\Delta_{o} f_{\nu, m, \lambda}^{k}=-k(k+1) f_{\nu, m, \lambda}^{k}$
(ii) $H_{3} f_{\nu, m, \lambda}^{k}=\nu f_{\nu, m, \lambda}^{k}$
(iii) $F_{+} f_{k, m, \lambda}^{k}=\sqrt{(2 k+1)(2 k+2)} C_{k+1, m} f_{k+1, m}^{k+1}$, where 


$$
C_{k+1, m}= \begin{cases}i \sqrt{\left\{(k+1)^{2}-\left(\frac{m}{2}\right)^{2}\right\}\left\{(k+1)^{2}+\frac{\lambda^{2}}{4}\right\} /\left\{4(k+1)^{2}-1\right\}} /(k+1) \\ i \sqrt{\left\{(k+1)^{2}+\lambda\right\} /\left\{4(k+1)^{2}-1\right\}} & \text { for } m=0\end{cases}
$$

(iv) Put $f_{\nu, m, \lambda}^{k}=0$ for $k=0,1 / 2,1,3 / 2, \cdots$ and $|\nu|=0,1 / 2,1, \cdots$ unless $\nu=-k,-k+1, \cdots, k$ and $k=m / 2, m / 2+1, \cdots$. Then the function $\left(T_{m, \lambda}(g) f_{\nu, m, \lambda}^{k}, f_{\nu^{\prime}, m, \lambda}^{k^{\prime}}\right)_{m, \lambda}$ on $G \times \hat{G}$ is measurable.

(v) As $t \rightarrow 0$, the norm

$$
\left\|\frac{T_{m, \lambda}\left(\omega_{j}(t)\right) f_{\nu, m, \lambda}^{k}-f_{\nu, m, \lambda}^{k}}{t}-\omega_{j} f_{\nu, m, \lambda}^{k}\right\|_{m, \lambda}
$$

converges to zero uniformly on any compact set of $\{(0, \lambda):-1<\lambda<0\}$, $\{(0, \lambda): \lambda \geqslant 0\}$ and $\ell_{m}$ with positive integer $m$.

Proof. A canonical basis has properties (i), (ii) and (iii). Assume that $g=\left(g_{i j}\right) \in G, \quad u \in S U(2), \quad\left(\begin{array}{cc}\alpha & \beta \\ -\bar{\beta} & \bar{\alpha}\end{array}\right) \in S U(2), \quad\left(\begin{array}{cc}\delta^{-1} & \mu \\ 0 & \delta\end{array}\right) \in K$ and that $\left(\begin{array}{cc}\alpha & \beta \\ -\bar{\beta} & \bar{\alpha}\end{array}\right) g=\left(\begin{array}{cc}\delta^{-1} & \mu \\ 0 & \delta\end{array}\right) u$, then we have (see $\S 11.1$ in [12])

$$
u_{22}=\left(-\bar{\beta} g_{12}+\bar{\alpha} g_{22}\right)\left\{\left|-\bar{\beta} g_{11}+\bar{\alpha} g_{21}\right|^{2}+\left|-\bar{\beta} g_{12}+\bar{\alpha} g_{22}\right|^{2}\right\}^{-1 / 2} .
$$

Hence $\alpha(u g) / \alpha(u \bar{g})$ is given by

$$
\begin{aligned}
& \left\{\left|-\bar{\beta} g_{11}+\bar{\alpha} g_{21}\right|^{2}+\left|-\bar{\beta} g_{12}+\bar{\alpha} g_{22}\right|^{2}\right\}^{-1+(i \rho-m) / 2} \quad \text { for } S_{m, \rho}, \\
& \left\{\left|-\bar{\beta} g_{11}+\bar{\alpha} g_{21}\right|^{2}+\left|-\bar{\beta} g_{12}+\bar{\alpha} g_{22}\right|^{2}\right\}^{-1-\sigma / 2} \quad \text { for } D_{\sigma} .
\end{aligned}
$$

Consequently $V(g) \varphi_{p, m, \rho}^{k}(u)$ and $V(g) \varphi_{p, \sigma}^{k}(u)$ are $C^{\infty}$-functions on $G \times S U(2)$ $\times \boldsymbol{R}$ and $G \times S U(2) \times(0,2)$ respectively. Recalling that the inner products of the representation space of $S_{m, \rho}$ and $D_{\sigma}$ are of the form

$$
\begin{aligned}
(\varphi, \varphi)_{m, \rho} & =\int_{S U(2)}|\varphi(u)|^{2} d u \\
(\varphi, \varphi)_{\sigma} & =\pi \iint_{S U(2) \times S U(2)} \Phi\left(u^{\prime} u^{\prime \prime-1}\right) \varphi\left(u^{\prime}\right) \overline{\varphi\left(u^{\prime \prime}\right)} d u^{\prime} d u^{\prime \prime}
\end{aligned}
$$

respectively, where $\Phi(u)=\left|u_{21}\right|^{-2+\sigma}$, we easily verify (iv). Since $V(g) \varphi(u)$ is smooth, (v) is clear.

Q.E.D.

Thanks to Lemma 2 (especially to (iv)), for a $\sigma$-finite measure on $G$ we can define a unitary representation $\int_{\hat{a}}^{\oplus} T_{m, \lambda} d \sigma$ on the Hilbert space 
$\int_{\hat{G}}^{\oplus} \mathfrak{S}_{m, \lambda} d \sigma$. To decompose a unitary representation of $G$ is, by definition, to determine a sequence of mutually singular $\sigma$-finite measures $\left\{\sigma_{1}, \sigma_{2}\right.$, $\left.\cdots, \sigma_{\infty}\right\}$ on the measurable space $\hat{G}$ so that the representation is unitarily equivalent to the representation $(T, H)$ defined by

$$
T=\int_{\hat{a}}^{\oplus} T_{m, \lambda} d \sigma_{1} \oplus[2] \int_{\hat{a}}^{\oplus} T_{m, \lambda} d \sigma_{2} \oplus \cdots \oplus\left[\boldsymbol{\aleph}_{0}\right] \int_{\hat{G}}^{\oplus} T_{m, \lambda} d \sigma_{\infty}
$$

on the Hilbert space

$$
\mathfrak{S}=\int_{\hat{a}}^{\oplus} \mathfrak{S}_{m, \lambda} d \sigma_{1} \oplus[2] \int_{\hat{a}}^{\oplus} \mathfrak{S}_{m, \lambda} d \sigma_{2} \oplus \cdots \oplus\left[\boldsymbol{K}_{0}\right] \int_{\hat{G}}^{\oplus} \mathfrak{S}_{m, \lambda} d \sigma_{\infty},
$$

where the cardinal number in the bracket indicates the multiplicity. We shall search for a procedure to determine the measure $\sigma_{i}$ up to the usual equivalence.

LEMma 3. For $k=0,1 / 2,1, \cdots$, let $W_{k}$ be the space of solutions of the equations

$$
H_{3} f=k f, \quad \Delta_{o} f=-k(k+1) f
$$

with respect to the representation $\left(T, \mathfrak{S}_{2}\right)$ above. Denote $\sigma_{i}^{(m)}$ the restriction $\sigma_{i} \mid \ell_{m}$. Then we have unitary equivalences among selfadjoint operators:

$$
\begin{array}{r}
\Delta \mid W_{0} \simeq \int_{[-1, \infty)}^{\oplus} \lambda d \sigma_{1}^{(0)} \oplus[2] \int_{[-1, \infty)}^{\oplus} \lambda d \sigma_{2}^{(0)} \oplus \cdots \oplus\left[\boldsymbol{K}_{0}\right] \int_{[-1, \infty)}^{\oplus} \lambda d \sigma_{\infty}^{(0)}, \\
\Delta^{\prime} \mid W_{k} \ominus F_{+} W_{k-1} \simeq \int_{R}^{\oplus}(-k) \lambda d \sigma_{1}^{(2 k)} \oplus[2] \int_{\boldsymbol{R}}^{\oplus}(-k) \lambda d \sigma_{2}^{(2 k)} \\
\oplus \cdots \oplus\left[\boldsymbol{K}_{0}\right] \int_{\boldsymbol{R}}^{\oplus}(-k) \lambda d \sigma_{\infty}^{(2 k)} .
\end{array}
$$

Proof. Without loss of generality we may assume that all measures except for $\sigma_{1}$ are zero measures. Rewrite $\sigma_{1}=\sigma$. We claim

$$
W_{k}=\left\{\int_{\hat{G}}^{\oplus} a(2 k, \lambda) f_{k, m, \lambda}^{k} d \sigma: \int_{\hat{G}}|a|^{2} d \sigma<\infty\right\}
$$

Indeed, set

$$
\tilde{W}_{k}=\left\{\int_{\hat{\sigma}}^{\oplus} \sum_{\nu=-k}^{k} a_{\nu}(m, \lambda) f_{\nu, m, \lambda}^{k} d \sigma: \int_{\hat{\sigma}}\left|a_{\nu}\right|^{2} d \sigma<\infty \text { for each } \nu\right\} .
$$

We will show that the restriction $\Delta_{o} \mid \tilde{W}_{k}$ is equal to $-k(k+1)$. To this end define $f(\varphi)$ for $f=\int_{\hat{a}}^{\oplus} f_{m, \lambda} d \sigma \in \tilde{W}_{k}$ and $\varphi$ in $C^{\infty}(S U(2))$ by $f(\varphi)=$ 
$\int_{S U(2)} \varphi(u) T(u) f d u \in \tilde{W}_{k}$. Denoting $\Delta_{o}^{r}$ and $\Delta_{o}^{m, \lambda}$ the operator $\Delta_{o}$ corresponding to the left regular representation of $S U(2)$ and the restriction $T_{m, 2} \mid S U(2)$ respectively, for $h=\int_{\hat{\sigma}}^{\oplus} h_{m, 2} d \sigma$ we have

$$
\begin{aligned}
\left(\Delta_{o} f(\varphi), h\right) & =\int_{S U(2)} d u\left(\Delta_{o}^{r} \varphi(u)\right)(T(u) f, h) \\
& =\int_{\hat{G}} d \sigma \int_{S U(2)} d u\left(\Delta_{o}^{r} \varphi(u)\right)\left(T_{m, \lambda}(u) f_{m, \lambda}, h_{m, \lambda}\right)_{m, \lambda} \\
& =\int_{\hat{G}} d \sigma\left(\Delta_{o}^{m, \lambda} f_{m, \lambda}(\varphi), h_{m, \lambda}\right)_{m, \lambda} \\
& =-k(k+1)(f(\varphi), h)
\end{aligned}
$$

as desired. Since the set $\left\{f_{\nu, m, \lambda}^{k}: \nu=-k,-k+1, \cdots, k\right.$ and $k=m / 2, m / 2$ $+1, \cdots\}$ is an orthonormal basis in the Hilbert space $\mathfrak{F}_{m, 2}, \mathfrak{F}$ is a direct sum of $\tilde{W}_{k}$ 's. Thus $W_{k}$ is a subspace of $\tilde{W}_{k}$. From (v) of Lemma $2 f=$ $\int_{\hat{a}}^{\oplus} \sum_{\nu=-k}^{k} a_{\nu}(m, \lambda) f_{\nu, m, \lambda}^{k} d \sigma$ in $\tilde{W}_{k}$ satisfies

$$
H_{3} f=\int_{\hat{a}}^{\oplus} \sum_{\nu=-k}^{k} \nu a_{\nu} f_{\nu, m, 2}^{k} d \sigma=k f,
$$

which implies that $a_{\nu}$ is equal to zero a.e. unless $\nu=k$, proving $1^{\circ}$. Next step is to show

$2^{\circ} \quad W_{k} \ominus F_{+} W_{k-1}=\left\{\int_{\ell_{2 k}}^{\oplus} a(2 k, \lambda) f_{k, 2 k, \lambda}^{k} d \sigma: \int_{\ell_{2 k}}|a|^{2} d \sigma<\infty\right\}$.

To see this, define $W_{k, m}=\left\{\int_{\ell_{m}}^{\oplus} a(m, \lambda) f_{k, m, \lambda}^{k} d \sigma: \int_{\ell_{m}}|a|^{2} d \sigma<\infty\right\}$. Since $W_{k}$ is a direct sum of $W_{k, m}$ 's with non-negative integers $m=2 k, 2 k-2, \cdots$ and since the closure $\overline{F_{+} W_{k-1, m}}$ coincides with $W_{k, m}$ due to (iii) and (v) of Lemma $2,2^{\circ}$ is now clear. Finally we verify $3^{\circ}$

$$
\begin{gathered}
\Delta \int_{\ell_{0}}^{\oplus} a(0, \lambda) f_{0,0, \lambda}^{0} d \sigma=\int_{\ell_{0}}^{\oplus} \lambda a(0, \lambda) f_{0,0, \lambda}^{0} d \sigma, \\
\Delta^{\prime} \int_{\ell_{2 k}}^{\oplus} a(2 k, \lambda) f_{k, 2 k, \lambda}^{k} d \sigma=\int_{\ell_{22 k}}^{\oplus}(-k) \lambda a(2 k, \lambda) f_{k, 2 k, \lambda}^{k} d \sigma,
\end{gathered}
$$

provided the members on the right side belong to $\mathfrak{F}$. Indeed we can argue as we showed that $\Delta_{o} \mid \tilde{W}_{k}=-k(k+1)$ in $1^{\circ}$. Now $1^{\circ}, 2^{\circ}$ and $3^{\circ}$ yield the Lemma.

Q.E.D.

The following lemma is also useful. 
LEMMA 4. The restriction $\Delta^{\prime} \mid W_{k}$ and $\Delta^{\prime} \mid \overline{F_{+} W_{k}}$ are unitarily equivalent selfadjoint operators.

Proof. As mentioned in the proof of Lemma 3, the closure $\overline{F_{+} W_{k}}$ is a direct sum of $W_{k+1, m}$ 's with non-negative integers $m=2 k, 2 k-2, \cdots$. The following isometry from $W_{k}$ onto $\overline{F_{+} W_{k}}$ transforms the first operator to the second one:

$$
\sum_{m=2 k, 2 k-2, \ldots} \int_{\ell_{m}}^{\oplus} a(m, \lambda) f_{k, m, \lambda}^{k} d \sigma \rightarrow \sum_{m=2 k, 2 k-2, \ldots} \int_{\ell_{m}}^{\oplus} a(m, \lambda) f_{k+1, m, \lambda}^{k+1} d \sigma .
$$

To sum up, given a unitary representation of $S L(2, C)$, one can decompose it into irreducible ones if one could specify the space $W_{k}$ (call it the space of the $k$-th heighest weight vectors) and carry out the spectral decomposition of selfadjoint operators $\Delta \mid W_{0}$ and $\Delta^{\prime} \mid W_{k} \ominus F_{+} W_{k-1}$.

\section{$\S 4$. The space of the k-th heighest weight vectors $W_{k}$}

Let $U^{i M, \pi}$ denote an irreducible unitary representation of the Poincaré group $P$ associated with the hyperboloid of one sheet $V_{i M}$ and an irreducible unitary representation $\pi$ of $S U(1,1)$ (see $\S 2$ ). In this section we shall first solve the equation (4), then determine the spectral type of selfadjoint operators $\Delta \mid W_{0}$ and $\Delta^{\prime} \mid W_{k}$ of the restriction $U^{i M, \pi} \mid S L(2, C)$. From now on $G$ and $G_{0}$ stand for $S L(2, C)$ and $S U(1,1)$ respectively.

We begin with specifying the representation $U^{i M, \pi}$ of $P . V_{i_{M}}=$ $\left\{y=\left(\begin{array}{ll}y_{0}-y_{3} & y_{2}-i y_{1} \\ y_{2}+i y_{1} & y_{0}+y_{3}\end{array}\right): \operatorname{det} y=-M^{2}\right\}$ in $\hat{R}_{4}$ is a $G$-homogeneous space with the invariant measure $d \mu(y)=d y_{1} d y_{2} d y_{3} /\left|y_{0}\right|$. Let $p$ be the projection from $G$ onto $V_{i M}$ defined by $p(g)=g^{*} \hat{x} g$, where $\hat{x}$ denotes the fixed point $M\left(\begin{array}{rr}1 & 0 \\ 0 & -1\end{array}\right)$. For $u$ in $S U(2)$ let $s_{u}$ be a measurable section from $V_{i M}$ into $G$ such that $p \circ s_{u}=$ identity and that

$$
s_{u} \circ p(\langle\tau, \theta, \varphi\rangle)=\langle\tau, \theta, \varphi\rangle u \quad \text { for }(\tau, \theta, \varphi) \in R \times(0, \pi) \times(0,2 \pi),
$$

where $\langle\tau, \theta, \varphi\rangle$ stands for the matrix $\omega_{6}(\tau) \omega_{2}(\theta) \omega_{3}(\varphi)$. We fix $s_{u}$ once for all. Then the representation $U^{i M, \pi}$ has the following realization $U^{\pi, u}$ on the Hilbert space $\mathfrak{S}^{\pi}=L^{2}\left(V_{i M}, \mathfrak{S}_{\pi}, \mu\right)$ for each $u \in S U(2)$ :

$$
\begin{gathered}
U^{\pi, u}(x, g) f(y)=e^{i\left\langle x^{\prime}, \hat{x}\right\rangle} \pi\left(g_{0}\right) f(y \cdot g), \\
s_{u}(y)(x, g)=\left(x^{\prime}, g_{0}\right) s_{u}(y \cdot g) \quad \text { with } g_{0} \in G_{0} .
\end{gathered}
$$


By the aid of the isometry $I_{u}: \widetilde{\mathfrak{F}}^{\pi}(G)=\left\{\tilde{f} \in L^{2}\left(G, \mathscr{S}_{\pi}, \mu\right): \tilde{f}\left(g_{0} g\right)=\pi\left(g_{0}\right) \tilde{f}(g)\right.$ for $\left.g_{0} \in G_{0}\right\} \rightarrow \mathscr{S}^{\pi}$ such that $\tilde{f}\left(s_{u}(y)\right)=I_{u} \tilde{f}(y), U^{\pi, u}$ is transformed to $U^{\pi, v}$ by $I_{v} I_{u}^{-1}$.

We proceed, assuming the representation $\pi$ to be $\pi_{(\ell, 0)}^{+}$. Other cases can be treated in the same way. Setting

$$
Y=\left\{p\left(\omega_{6}(\tau) \omega_{2}(\theta) \omega_{3}(\varphi)\right):(\tau, \theta, \varphi) \in R \times(0, \pi) \times(0,2 \pi)\right\} \subset V_{i M},
$$

for $u \in S U(2)$ define a dense subspace $\mathfrak{S C}_{0}^{\pi, u}$ of $\mathfrak{S C}^{\pi}$ :

$$
\mathfrak{S}_{\mathcal{2}}^{\pi, u}=\left\{f \in C_{0}^{\infty}(Y \cdot u \times T): f\left(y, e^{i \psi}\right)=\sum_{\nu \geqslant-\ell} f_{\nu}(y) e^{i \nu \psi}\right\} .
$$

We note that for $f$ in $\mathfrak{S}_{\mathcal{C}_{0}^{\pi}, u}(6)$ takes the form

$$
U^{\pi, u}(0, g) f\left(y, e^{i \psi}\right)=\left|\beta e^{i \psi}+\bar{\alpha}\right|^{2 \ell} f\left(y \cdot g, \frac{\alpha e^{i \psi}+\bar{\beta}}{\beta e^{i \psi}+\bar{\alpha}}\right)
$$

provided $s_{u}(y) g=g_{0} s_{u}(y \cdot g)$ with $g_{0}=\left(\begin{array}{ll}\frac{\alpha}{\beta} & \frac{\beta}{\alpha}\end{array}\right) \in G_{0}$. Since the section $s_{u}$ is smooth on $Y \cdot u$ as well as the map $(y, g) \rightarrow y \cdot g$, there exists a relatively compact neighborhood $U$ of the unit element of $G$ such that for $f \in \mathfrak{F}_{2}^{\pi, u}$, the function $U^{\pi, u}(0, g) f\left(y, e^{i \psi}\right)$ belong to $C^{\infty}(U \times Y \cdot u \times T)$. This observation leads to

LeMma 5. The domain of $\omega_{j}^{\pi, u}$ includes $\mathfrak{S}_{0}^{\pi}, u$ for all $j$ and the restriction $\omega_{j}^{\pi, u} \mid \mathcal{S}_{0}^{\pi, u}$ is a differential operator with $C^{\infty}$-coefficients.

Now that $\omega_{j}^{\pi, u}$ is a continuous transformation of $S_{2}{ }^{\pi, u}$ with the relative topology of $C_{0}^{\infty}(Y \cdot u \times T)$, we define the dual operator $\hat{\omega}_{j}^{\pi, u}$ by the following

$$
\left\langle\hat{\omega}_{j}^{\pi, u} \hat{f}, f\right\rangle=\left\langle\hat{f}, \omega_{j}^{\pi, u} f\right\rangle
$$

where $\hat{f} \in\left(\mathfrak{S}_{0}^{\pi}, u\right)^{\prime}$ and $f \in \mathfrak{S C}_{0}^{\pi}, u$. Regarding $\mathfrak{S}^{\pi}$ as a subspace of the dual space $\left(\mathfrak{S}_{0}^{\pi, u}\right)^{\prime}$, we claim

Lemma 6.

(i ) $\omega_{j}^{\pi, u} \subset-\hat{\omega}_{j}^{\pi, u}$.

(ii) Assume that $f$ belongs to $\mathfrak{S}_{0}^{\pi, u}$ and $\operatorname{Supp} f \subset Y \cdot v$ for some $v \in S U(2)$. Then $f^{v}=I_{v} I_{u}^{-1} f$ belongs to $\mathfrak{S}_{0}^{\pi, v}$ and satisfies

$$
\left(\omega_{j}^{\pi, u} f, h\right)=\left(\omega_{j}^{\pi, v} f^{v}, h^{v}\right) \quad \text { for any } h \in \mathfrak{S}^{\pi} .
$$

(iii) The intersection $D_{\Delta_{0}^{\pi}, u} \cap D_{\Delta^{\pi}, u} \cap D_{4^{\prime} \pi, u}$ includes $\mathfrak{F}_{0}^{\pi, u}$. Further- 
more, it holds that (the indexes $\pi$ and $u$ are omitted)

$$
\begin{aligned}
& \Delta_{o} \subset \sum_{i=1}^{3}\left(\hat{\omega}_{j}\right)^{2}, \quad \Delta \subset \sum_{i=1}^{3}\left(\hat{\omega}_{i}\right)^{2}-\sum_{j=4}^{6}\left(\hat{\omega}_{j}\right)^{2}-1, \\
& \Delta^{\prime} \subset-\left(\hat{\omega}_{1} \hat{\omega}_{4}+\hat{\omega}_{4} \hat{\omega}_{1}+\hat{\omega}_{2} \hat{\omega}_{5}+\hat{\omega}_{5} \hat{\omega}_{2}+2 \hat{\omega}_{3} \hat{\omega}_{6}\right) .
\end{aligned}
$$

Proof. Since $\omega_{j}^{\pi, u}$ is antihermitian, (i) follows. We note that $f^{v}(y)$ $=\pi\left(g_{0}\right) f(y)$ provided $s_{v}(y)=g_{0} s_{u}(y)$ with $g_{0}=\left(\begin{array}{cc}\alpha & \beta \\ \beta & \frac{\beta}{\alpha}\end{array}\right) \in G_{0}$, namely

$$
f^{v}\left(y, e^{i \psi}\right)=\left|\beta e^{i \psi}+\bar{\alpha}\right|^{2 \ell} f\left(y, \frac{\alpha e^{i \psi}+\bar{\beta}}{\beta e^{i \psi}+\bar{\alpha}}\right) .
$$

Since $g_{0}$ is smooth on $Y \cdot u \cap Y \cdot v, f^{v}$ has a representative in $\mathfrak{S}_{0}^{\pi, v}$. Now (ii) is evident. As to (iii) we deal only with $\Delta^{x, u}$. It suffices to prove

$$
\begin{aligned}
& \Delta^{\pi, u} \int_{G} \varphi(g) U^{\pi, u}(0, g) f d g \\
& \quad=\int_{G} \varphi(g) U^{\pi, u}(0, g)\left[\sum_{i}\left(\omega_{i}^{\pi, u}\right)^{2}-\sum_{j}\left(\omega_{j}^{\pi, u}\right)^{2}-1\right] f d g
\end{aligned}
$$

for $\varphi \in C_{0}^{\infty}(G)$ and $f \in \mathfrak{S}_{0}^{\pi, u}$ [14]. To this end we will show that for $\psi \in C_{0}^{\infty}(G)$ and $h \in \mathfrak{S}_{0}^{\pi}, u$

$$
\begin{aligned}
& \left(\Delta^{\pi, u} \int \varphi(g) U^{\pi, u}(0, g) f d g, \int \psi\left(g^{\prime}\right) U^{\pi, u}\left(0, g^{\prime}\right) h d g^{\prime}\right) \\
& \quad=\left(\int \varphi(g) U^{\pi, u}(g)\left[\sum_{i}\left(\omega_{i}^{\pi, u}\right)^{2}-\sum_{j}\left(\omega_{j}^{\pi, u}\right)^{2}-1\right] f d g\right. \\
& \left.\int \psi\left(g^{\prime}\right) U^{\pi, u}\left(0, g^{\prime}\right) h d g^{\prime}\right) .
\end{aligned}
$$

A diffeomorphism $q: V_{i M} \rightarrow \boldsymbol{R} \times S_{2}$ defined by

$$
q(y)=\left(y_{0}, y_{1} /\left(\sqrt{y_{1}^{2}+y_{2}^{2}+y_{3}^{2}}, \quad y_{2} / \sqrt{y_{1}^{2}+y_{2}^{2}+y_{3}^{2}}, \quad y_{3} / \sqrt{\left.y_{1}^{2}+y_{2}^{2}+y_{3}^{2}\right)}\right)\right.
$$

maps $Y \cdot u$ onto $R \times S_{2}^{u}$. We note that each $S_{2}^{u}$ is dense and open in the unit sphere $S_{2}$ and that the union $\cup_{u \in S U(2)} S_{2}^{u}$ covers the sphere. Observing that for given $a, a^{\prime} \in G$ and $y, y^{\prime} \in V_{i M}$ there exists $w \in S U(2)$ such that $\left\{y, y^{\prime}, y^{\prime} \cdot a^{\prime-1} a\right\} \subset Y \cdot w$, we can show inductively that there exist a finite covering $\left\{U_{\alpha}\right\}$ of $\operatorname{Supp} \varphi$, finite covering $\left\{U_{\alpha \beta}\right\}$ of $\operatorname{Supp} \psi$, finite covering $\left\{Y_{\alpha \beta r}\right\}$ of $\operatorname{Supp} f$, finite covering $\left\{Y_{\alpha \beta \gamma \delta}\right\}$ of $\operatorname{Supp} h$ and $w_{\alpha \beta \gamma \delta} \in S U(2)$ such that each member is relatively compact and that

$$
Y_{\alpha \beta \gamma} \cup Y_{\alpha \beta \gamma \delta} \cup Y_{\alpha \beta \gamma \delta} \cdot U_{\alpha \beta}^{-1} U_{\alpha} \subset Y \cdot w .
$$


Denote $\chi_{\alpha}, \chi_{\alpha \beta}, \chi_{\alpha \beta r}$ and $\chi_{\alpha \beta \gamma \delta}$ the partition of unity associated with the coverings above. Now the left side of (9) is equal to

$$
\begin{aligned}
\int d g \varphi(g) & \left(f, U^{\pi, u}\left(g^{-1}\right) \Delta^{\pi, u} \int \psi\left(g^{\prime}\right) U^{\pi, u}\left(g^{\prime}\right) h d g^{\prime}\right) \\
= & \int d g \varphi(g)\left(f, \Delta^{\pi, u} U^{\pi, u}\left(g^{-1}\right) \int \psi\left(g^{\prime}\right) U^{\pi, u}\left(g^{\prime}\right) h d g^{\prime}\right) \\
& =\int d g \varphi(g)\left(f, \Delta^{\pi, u} \int \psi\left(g^{\prime}\right) U^{\pi, u}\left(g^{-1} g^{\prime}\right) d g^{\prime}\right) \\
& =\int \sum_{\alpha, \beta, r, \delta} \int d g \varphi \chi_{\alpha}\left(f \chi_{\alpha \beta r}, \Delta^{\pi, u} \int \psi \chi_{\alpha \beta} U^{\pi, u}\left(g^{-1} g^{\prime}\right) h \chi_{\alpha \beta \gamma \delta} d g^{\prime}\right) .
\end{aligned}
$$

Putting $w=w_{\alpha \beta \gamma \delta}$ we rewrite the $\alpha \beta \gamma \delta$-term above as

$$
\int d g \varphi \chi_{\alpha}\left(\left(f \chi_{\alpha \beta \gamma}\right)^{w}, \Delta^{\pi, w} \int \psi \chi_{\alpha \beta} U^{\pi, w}\left(g^{-1} g^{\prime}\right)\left(h \chi_{\alpha \beta \gamma \delta}\right)^{w} d g^{\prime}\right) \text {. }
$$

Since $\chi_{\alpha}(g) \int \psi \chi_{\alpha \beta} U^{\pi, w}\left(h \chi_{\alpha \beta \gamma \partial}\right)^{w} d g^{\prime}$ belongs to $\mathfrak{S}_{0}^{\pi, w}$, it holds that

$$
\begin{aligned}
& \Delta^{\pi, w} \chi_{\alpha}(g) \int \psi \chi_{\alpha \beta} U^{\pi, w}\left(h \chi_{\alpha \beta \gamma \delta}\right)^{w} d g^{\prime} \\
& \quad=\chi_{\alpha}(g)\left[\sum_{i}\left(\omega_{i}^{\pi, w}\right)^{2}-\sum_{j}\left(\omega_{j}^{\pi, w}\right)^{2}-1\right] \int \psi \chi_{\alpha \beta} U^{\pi, w}\left(h \chi_{\alpha \beta \gamma \delta}\right)^{w} d g^{\prime} .
\end{aligned}
$$

On account of Lemma 5 and (ii) of Lemma 6 the $\alpha \beta \gamma \delta$-term is equal to

$$
\int d g \varphi \chi_{\alpha}\left(\left[\sum_{i}\left(\omega_{i}^{\pi, u}\right)^{2}-\sum_{j}\left(\omega_{j}^{\pi, u}\right)^{2}-1\right] f \chi_{\alpha \beta r}, \int \psi \chi_{\alpha \beta} U^{\pi, u}\left(h \chi_{\alpha \beta \gamma \delta}\right) d g^{\prime}\right),
$$

from which (9) follows.

Q.E.D.

We now derive the concrete forms of the restrictions to $\mathfrak{S}_{0}^{\pi, e}$ of $\omega_{i}, H_{i}, F_{i}, \Delta_{o}, \Delta$ and $\Delta^{\prime}$ with respect to the representation $\left(U^{\pi, e}, \mathfrak{S}^{\pi}\right)$. After tedious computation we obtain the following. The underlined terms disappear for nonspinor irreducible unitary representations $\pi_{(\ell, 0)}$ and $\pi_{(\ell, 0)}^{ \pm}$of $S U(1,1)$.

$$
\begin{gathered}
p\left(\omega_{6}(\tau) \omega_{2}(\theta) \omega_{3}(\varphi)\right)=\left(\begin{array}{cc}
-e^{\tau} \cos ^{2} \theta / 2+e^{-\tau} \sin ^{2} \theta / 2 & \operatorname{ch} \tau \sin \theta e^{-i \varphi} \\
\operatorname{ch} \tau \sin \theta e^{i \varphi} & -e^{\tau} \sin ^{2} \theta / 2+e^{-\tau} \cos ^{2} \theta / 2
\end{array}\right), \\
\left(y_{0}, y_{1}, y_{2}, y_{3}\right)=(-\operatorname{sh} \tau, \operatorname{ch} \tau \sin \theta \sin \psi, \operatorname{ch} \tau \sin \theta \cos \varphi, \operatorname{ch} \tau \cos \theta), \\
d \mu=\operatorname{ch}^{2} \tau \sin \theta d \tau d \theta d \varphi, \\
\omega_{1}=\sin \varphi \partial_{\theta}+\cot \theta \cos \varphi \partial_{\varphi}-\frac{\cos \varphi}{\sin \theta} \partial_{\psi}+\frac{i \cos \varphi}{2 \sin \theta},
\end{gathered}
$$




$$
\begin{aligned}
& \omega_{2}=\cos \varphi \partial_{\theta}-\cot \theta \sin \varphi \partial_{\varphi}+\frac{\sin \varphi}{\sin \theta} \partial_{\psi}-\frac{i \sin \varphi}{2 \sin \theta}, \\
& \omega_{3}=\partial_{\varphi}, \\
& \omega_{4}=-\sin \theta \cos \varphi \partial_{\tau}-\operatorname{th} \tau \cos \theta \cos \varphi \partial_{\theta}+\frac{\operatorname{th} \tau \sin \varphi}{\sin \theta} \partial_{\varphi} \\
& +\left(-\operatorname{th} \tau \cot \theta \sin \varphi-\frac{\cos \theta \cos \varphi \sin \psi+\sin \varphi \cos \psi}{\operatorname{ch} \tau}\right) \partial_{\psi} \\
& +\frac{\ell(\cos \theta \cos \varphi \cos \psi-\sin \varphi \sin \psi)}{\operatorname{ch} \tau} \\
& +\frac{i(\cos \theta \cos \varphi \sin \psi+\sin \varphi \cos \psi)}{2 \operatorname{ch} \tau}+\frac{\operatorname{th} \tau \cot \theta \sin \varphi}{2}, \\
& \omega_{5}=\sin \theta \sin \varphi \partial_{\tau}+\operatorname{th} \tau \cos \theta \sin \varphi \partial_{\theta}+\frac{\operatorname{th} \tau \cos \varphi}{\sin \theta} \partial_{\varphi} \\
& +\left(-\operatorname{th} \tau \cot \theta \cos \varphi+\frac{\cos \theta \sin \varphi \sin \psi-\cos \varphi \cos \psi}{\operatorname{ch} \tau}\right) \partial_{\psi} \\
& +\frac{\ell(-\cos \theta \sin \varphi \cos \psi-\cos \varphi \sin \psi)}{\operatorname{ch} \tau} \\
& +\frac{i(-\cos \theta \sin \varphi \sin \psi+\cos \varphi \cos \psi)}{2 \operatorname{ch} \tau}+\frac{\operatorname{th} \tau \cot \theta \cos \varphi}{2}, \\
& \omega_{6}=\cos \theta \partial_{\tau}-\operatorname{th} \tau \sin \theta \partial_{\theta}-\frac{\sin \theta \sin \psi}{\operatorname{ch} \tau} \partial_{\psi}+\frac{\ell \sin \theta \cos \psi}{\operatorname{ch} \tau} \\
& +\frac{i \sin \theta \sin \psi}{2 \operatorname{ch} \tau} \\
& H_{+}=e^{-i \varphi}\left(i \partial_{\theta}+\cot \theta \partial_{\varphi}-\frac{1}{\sin \theta} \partial_{\psi}+\frac{i}{2 \sin \theta}\right), \\
& H_{-}=e^{+i \varphi}\left(i \partial_{\theta}-\cot \theta \partial_{\varphi}+\frac{1}{\sin \theta} \partial_{\psi}-\frac{i}{2 \sin \theta}\right), \\
& H_{3}=i \partial_{\varphi}, \\
& F_{+}=e^{-i \varphi}\left[-\sin \theta \partial_{\tau}-\operatorname{th} \tau \cos \theta \partial_{\theta}+\frac{i \operatorname{th} \tau}{\sin \theta} \partial_{\varphi}\right. \\
& +\left(-i \operatorname{th} \tau \cot \theta-\frac{\cos \theta \sin \psi+i \cos \psi}{\operatorname{ch} \tau}\right) \partial_{\psi} \\
& +\frac{\ell(\cos \theta \cos \psi-i \sin \psi)}{\operatorname{ch} \tau}+\frac{i \cos \theta \sin \psi-\cos \psi}{2 \operatorname{ch} \tau} \\
& \left.-\frac{\operatorname{th} \tau \cot \theta}{2}\right] \text {, }
\end{aligned}
$$




$$
\begin{aligned}
& F_{-}=e^{i \varphi}\left[\sin \theta \partial_{\tau}+\operatorname{th} \tau \cos \theta \partial_{\theta}+\frac{i \text { th } \tau}{\sin \theta} \partial_{\varphi}+(-i \text { th } \tau \cot \theta\right. \\
& \left.+\frac{\cos \theta \sin \psi-i \cos \psi}{\operatorname{ch} \tau}\right) \partial_{\psi}-\frac{i \cos \theta \sin \psi+\cos \psi}{2 \operatorname{ch} \tau} \\
& \left.-\frac{\operatorname{th} \tau \cot \theta}{2}+\frac{\ell(-\cos \theta \cos \psi-i \sin \psi)}{\operatorname{ch} \tau}\right] \text {, } \\
& F_{3}=i\left[\cos \theta \partial_{\tau}-\operatorname{th} \tau \sin \theta \partial_{\theta}-\frac{\sin \theta \sin \psi}{\operatorname{ch} \tau} \partial_{\psi}+\frac{\ell \sin \theta \cos \psi}{\operatorname{ch} \tau}\right. \\
& \left.+\frac{i \sin \theta \sin \psi}{2 \operatorname{ch} \tau}\right] \\
& \Delta_{0}=\partial_{\theta}^{2}+\frac{1}{\sin ^{2} \theta} \partial_{\varphi}^{2}-\frac{2 \cot \theta}{\sin \theta} \partial_{\varphi} \partial_{\psi}+\frac{1}{\sin ^{2} \theta} \partial_{\psi}^{2}+\cot \theta \partial_{\theta} \\
& +\frac{i \cot \theta}{2 \sin \theta} \partial_{\varphi}-\frac{i}{2 \sin ^{2} \theta} \partial_{\psi}-\frac{1}{4 \sin ^{2} \theta}, \\
& \Delta^{\prime}=-2 \partial_{\tau} \partial_{\psi}+\frac{2 \cos \psi}{\operatorname{ch} \tau} \partial_{\theta} \partial_{\psi}+\frac{2 \sin \psi}{\operatorname{ch} \tau \sin \theta} \partial_{\varphi} \partial_{\psi}-\frac{2 \cot \theta \sin \psi}{\operatorname{ch} \tau} \partial_{\psi}^{2}+i \partial_{\tau} \\
& +\left(\frac{\ell \sin \psi}{\operatorname{ch} \tau}-\frac{i \cos \psi}{\operatorname{ch} \tau}\right) \partial_{\theta}+\left(-\frac{2 \ell \cos \psi}{\operatorname{ch} \tau \sin \theta}-\frac{i \sin \psi}{\operatorname{ch} \tau \sin \theta}\right) \partial_{\varphi} \\
& +2\left(\frac{\ell \cot \theta \cos \psi}{\operatorname{ch} \tau}-\operatorname{th} \tau+\frac{i \cot \theta \sin \psi}{\operatorname{ch} \tau}\right) \partial_{\psi} \\
& +\left(-\frac{i \ell \cot \theta \cos \psi}{\operatorname{ch} \tau}+\frac{\cot \theta \sin \psi}{\operatorname{ch} \tau}+i \operatorname{th} \tau\right) \text {, } \\
& \Delta=-\left(\partial_{\tau}^{2}+2 \operatorname{th} \tau \partial_{\tau}+\frac{\ell(\ell+1)}{\operatorname{ch}^{2} \tau}+1\right)+S \text {. }
\end{aligned}
$$

We remark that the differential operator $S$ does not contain any terms of the form $S(\tau, \theta, \varphi, \psi) \partial_{\tau}^{j}(j=0,1,2)$.

We are ready to solve the equation (4). Consider the following equation

$$
-i \hat{\omega}_{3} f=k f, \quad \sum_{i=1}^{3} \hat{\omega}_{i}^{2} f=-k(k+1) f, \quad f \in \mathfrak{S C}^{\pi}(k=-\ell,-\ell+1, \cdots)
$$

and denote $\hat{W}_{k}$ the space of solutions (in (11) we omitted the indexes $\pi$ and $e$ for the sake of simplicity). Lemma 6 implies that $W_{k}$ is the intersection of $\hat{W}_{k}, D_{H_{3}}$ and $D_{\Delta_{0}}$.

LEMMA 7. An $\hat{f}$ belongs to $\hat{W}_{k}$ if and only if $f$ is of the form:

$$
\hat{f}\left(\tau, \theta, \varphi, e^{i \psi}\right)=\sum_{\nu \geqslant-\ell}^{k} \sum_{i=1,2} f_{\nu, i}(\tau) Q_{\nu, i}(\cos \theta) e^{-i k \varphi+i \nu \psi},
$$


where $f_{\nu, i}$ belongs to $L^{2}\left(R, \operatorname{ch}^{2} \tau d \tau\right)$ and $\left\{Q_{\nu, i}(z): i=1,2\right\}$ span the space of solutions in $L^{2}((-1,1))$ of the equation:

$$
\left[\left(1-z^{2}\right) \partial_{z}^{2}-2 z \partial_{z}-\frac{k^{2}+\nu^{2}+2 k \nu z}{1-z^{2}}+k(k+1)\right] Q(z)=0 \text { on }(-1,1) .
$$

For the proof we need

Lemma 8. Assume that $k$ ranges $0,1 / 2,1, \cdots$ and that $k+\nu$ is an integer. Then the equation (13) has no solutions in $L^{2}$ for $|\nu|>k$, while the bounded solution of (13) is proportional to $P_{k,-\nu}^{k}(z)$ for $|\nu| \leqslant k$. $P_{k, \nu}^{k}$ is defined by

$$
P_{k, \nu}^{k}(z)=\frac{i^{k-\nu}}{2^{k}} \sqrt{\frac{(2 k) !}{(k-\nu) !(k+\nu) !}}(1-z)^{(k-\nu) / 2}(1+z)^{(k+\nu) / 2} .
$$

Proof of Lemma 8. A similar statement can be found in chap. 3, sec. 4 [17]. That $P_{k,-\nu}^{k}$ is a bounded solution of (13) is known. By the change of variable $t=(z+1) / 2$, the solution of (13) may be written as

$$
\begin{aligned}
& P\left(\begin{array}{cccc}
-1 & 1 & \infty & \\
-|k-\nu| / 2 & -|k+\nu| / 2 & -k & z \\
|k-\nu| / 2 & |k+\nu| / 2 & k+1
\end{array}\right)=P\left(\begin{array}{cccc}
-1 & 1 & \infty & \\
\alpha & \gamma & \beta & z \\
\alpha^{\prime} & \gamma^{\prime} & \beta^{\prime} &
\end{array}\right)
\end{aligned}
$$

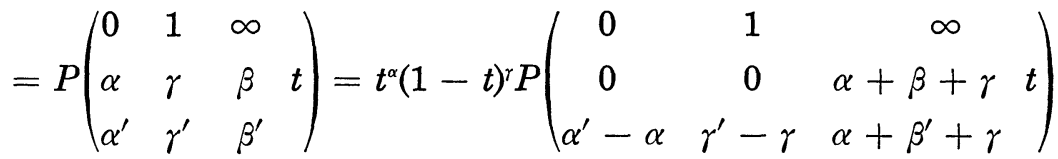

$$
\begin{aligned}
& =t^{\alpha}(1-t)^{r} P\left(\begin{array}{cccc}
0 & 1 & \infty & \\
0 & 0 & a & t \\
1-c & c-a-b & b
\end{array}\right) \text {. }
\end{aligned}
$$

If $c<1$, equivalently $k \neq \nu$, then $t^{\alpha}(1-t)^{r} F(a, b, a+b-c, 1-t)$ and $t^{a}(1-t)^{c-a-b} F(c-a, c-b, c-a-b+1,1-t)$ are linearly independent solutions around $t=1$, where $F(a, b, c, t)$ denotes the hypergeometric function. Checking the behavior of them around $t=0$ and 1 [5], one verifies the lemma for $k \neq \nu$. If $c=1, w_{1}=P_{k,-k}^{k}$ is a solution. As is well known, a linearly independent solution $w_{2}$ has the form

$$
c_{-1} w_{1}(z) \log (z+1)+\sum_{n=0} c_{n}(z+1)^{n} \quad \text { with } c_{-1} c_{0} \neq 0 .
$$

This function is unbounded around $z=-1$.

Q.E.D. 
Proof of Lemma 7. Expand $\hat{f}: \hat{f}\left(y, e^{i \psi}\right)=\sum_{\nu \geqslant-\ell} \hat{f}_{\nu}(y) e^{i \nu \psi}$. For $h(\tau, \theta, \varphi, \psi)$ $=h_{1}(\tau) h_{2}(\theta) h_{3}(\varphi) e^{i \nu \psi}$ with $h_{i} \in C_{0}^{\infty}$ we have

$$
\left(-i \hat{f}, \omega_{3} h\right)=k(\hat{f}, h),
$$

from which it follows that $\hat{f}_{\nu}(y)$ is of the form $f_{\nu}(\tau, \theta) e^{-i k \varphi}$ with $f_{\nu} \in L^{2}(R$ $\left.\times(0, \pi): \operatorname{ch}^{2} \tau \sin \theta d \tau d \theta\right)$. Furthermore $f$ satisfies

$$
\begin{aligned}
0= & \left(f,\left[\Delta_{o}+k(k+1)\right] h\right)=\left(f,\left[\partial_{\theta}^{2}+\cot \theta \partial_{\theta}+\frac{1}{\sin ^{2} \theta} \partial_{\varphi}^{2}-\frac{2 \nu \cot \theta}{\sin \theta} \partial_{\varphi}\right.\right. \\
& \left.\left.-\frac{\nu^{2}}{\sin ^{2} \theta}+k(k+1)\right] h\right) \\
= & \left\|e^{i \nu \psi}\right\|^{2}\left(e^{-i k \varphi}, h_{3}\right)\left(f_{v},\left[\partial_{\theta}^{2}+\cot \theta \partial_{\theta}-\frac{k^{2}+\nu^{2}+2 k \nu \cos \theta}{\sin ^{2} \theta}\right.\right. \\
& \left.+k(k+1)] h_{1} h_{2}\right) .
\end{aligned}
$$

Putting $G_{\nu}(\tau, \cos \theta)=f_{\nu}(\tau, \theta)$, we conclude that $G_{\nu}(\tau, z)$ is a weak solution, consequently, a smooth solution of (13) for a.e. $\tau$. Thus $f$ must have the desired expression. Conversely if $f$ is of the form (10), it satisfies (11) because $h$ 's finite linear combinations form a dense set in $\mathfrak{S}_{0}^{\pi, e}$. Q.E.D.

Lemma 9. Assume $f$ in $\mathfrak{S}^{\pi}$ to be of the form

$$
f\left(\tau, \theta, \varphi, e^{i \psi}\right)=\sum_{\nu \geqslant-\ell}^{k} f_{\nu}(\tau) P_{k,-\nu}^{k}(\cos \theta) e^{-i k \varphi+i \nu \psi}
$$

for some integer $k$ and $f_{\nu}$ in $C_{0}^{\infty}(R)$. Then $f$ belongs to domains of $\omega_{j}, \Delta_{o}, \Delta$ and $\Delta^{\prime}(j=1,2, \cdots, 6)$. $F$ belongs to $W_{k}$, too.

Proof. We may suppose $f=f_{\nu} P_{k,-\nu}^{k} e^{-i k \varphi+i \nu \psi}$. We will show that there exists an $\tilde{f}$ in $\mathfrak{S C}^{\pi}(G)$ such that

$$
\tilde{f}\left(\omega_{\theta}(\tau) u, e^{i \psi}\right)=f_{\nu}(\tau) t_{-\nu,-k}^{k}(u) e^{i \nu \psi}, \quad I_{e} \tilde{f}=f
$$

(see below (7) for the definition of $\mathfrak{S C}^{\pi}(G)$ and $I_{e}$ ), where $t_{m, n}^{t}(u)$ is the $(m, n)$ matrix element corresponding to an irreducible unitary representation of $S U(2)$ (chap. 3 [17]). It suffices to prove

$$
f_{\nu}\left(\tau^{\prime}\right) t_{-\nu,-k}^{k}\left(u^{\prime}\right) e^{i \nu \psi}=\pi\left(g_{0}\right)\left(f_{\nu}(\tau) t_{-\nu,-k}^{k}(u) e^{i \nu \psi}\right)
$$

assuming that $\omega_{6}\left(\tau^{\prime}\right) u^{\prime}=g_{0} \omega_{6}(\tau) u$. As one verifies easily, the condition implies that $\tau^{\prime}=\tau$ and $g_{0}=\omega_{3}(t)$ for some $t$. Thus it holds that

$$
t_{-,-k}^{k}\left(u^{\prime}\right)=e^{i \nu t} t_{-\nu,-k}^{k}(u), \quad \pi\left(g_{0}\right) e^{i \nu \psi}=e^{i \nu(t+\psi)},
$$


which proves (16). Take a compact set $B$ of the hyperboloid $V_{i M}$ so that any $f \circ s_{u}(u \in S U(2))$ vanishes on the complement $B^{c}$, then find a finite covering $\left\{Y_{\alpha}\right\}$, the partition of unity and a finite set $\left\{u_{\alpha}\right\} \subset S U(2)$ satisfying Supp $\chi_{\alpha} \subset Y \cdot u_{\alpha}$. Since $I_{u_{\alpha}} I_{e}^{-1} f \chi_{\alpha}=\left(\tilde{f} \cdot s_{u_{\alpha}}\right) \chi_{\alpha}$ belongs to $\mathfrak{S}_{0}^{\pi, u_{\alpha}}, D_{\Delta \pi, u_{\alpha}}$, for example, contains it due to Lemma 6. This in turn implies that $f \chi_{\alpha}$, hence $f$ itself, belongs to the domain of $\Delta^{\pi, e}$. Recalling $W_{k}=\hat{W}_{k} \cap D_{H_{3}}$ $\cap D_{\Delta_{0}}$, we complete the proof.

Q.E.D.

Finally we solve the equations (4).

Proposition 1. The space of $k$-th heighest weight vectors $W_{k}$ for the representation $U^{\pi, e} \mid S L(2, C)$ with $\pi=\pi_{(\ell, 0)}^{+}$is as follows:

$$
\begin{aligned}
& W_{k}=\left\{\sum_{\nu \geqslant-\ell}^{k} f_{\nu}(\tau) P_{k,-\nu}^{k}(\cos \theta) e^{-i k \varphi+i \nu \psi}: f_{\nu} \in L^{2}\left(R, \operatorname{ch}^{2} d \tau\right)\right\} \\
& =\{0\} \quad \text { otherwise. }
\end{aligned}
$$

Proof. Since $U^{\pi, e}(0,-e)=I, W_{k}$ is a null space provided $k$ is a half integer. On account of Lemma 9 and closedness of $H_{3}$ and $\Delta_{o}, W_{k}$ includes the right side above. Keeping Lemma 7 in mind and assuming that

$$
f\left(\tau, \theta, \varphi, e^{i \psi}\right)=\sum_{\nu \geqslant-\ell}^{k} f_{\nu}(\tau) Q_{\nu}(\cos \theta) e^{-i k \varphi+i \nu \psi},
$$

where $Q_{\nu}(z)$ is a $L^{2}$-solution of (13) which is independent of $P_{k,-\nu}^{k}(z)$, we will show the opposite inclusion. By Lemma $8, Q_{\nu}$ is either identically zero or unbounded arround -1 or 1 . From (8) we see that $f^{u}=I_{u} \circ I_{e}^{-1} f$ has the form:

$$
f^{u}\left(\tau, \theta, \varphi, e^{i \psi}\right)=\sum_{\nu \geqslant-\ell}^{k} f_{\nu}(\tau) Q_{\nu}\left(\cos \theta^{\prime}\right) e^{-i k \varphi^{\prime}+i \nu t+i \nu \psi}
$$

provided $\omega_{6}(\tau) \omega_{2}(\theta) \omega_{3}(\varphi) u=\omega_{6}\left(\tau^{\prime}\right) \omega_{3}(t) \omega_{2}\left(\theta^{\prime}\right) \omega_{3}\left(\varphi^{\prime}\right)$. Since $f^{u}$ belongs to $\hat{W}_{k}^{\pi, u}$, it satisfies

$$
\sum_{i=1}^{3}\left(\hat{\omega}_{i}^{\pi}, u\right)^{2} f^{u}=-k(k+1) f^{u} .
$$

Put $Q_{\nu}^{u}(\theta, \varphi)=Q_{\nu}\left(\cos \theta^{\prime}\right) e^{i k \varphi^{\prime}+i \nu t}$. Assume that $Q_{\nu}(z)$ is unbounded around 1 and that for a positive constant a $a^{-1}<\left|f_{\nu}(\tau)\right|<a$ on a non-null set $B_{\nu}$. In other words we assume that $f_{\nu}(\tau) Q_{\nu}(\cos \theta) e^{-i k \varphi}$, as a function on $Y$, is not essentially bounded around $y=(-\operatorname{sh} \tau, 0,0,1)$. Let $u \in S U(2)$ be so chosen that $q \circ p\left(\omega_{6}(\tau) \omega_{1}(\pi / 2) \omega_{3}(\pi) u\right)=y$ (see (10) for $q$ ). By the assumption 
$f_{\nu}(\tau) Q_{\nu}^{u}(\theta, \varphi)$ is not essentially bounded on $B_{\nu} \times(\pi / 2-\varepsilon, \pi / 2+\varepsilon) \times(\pi-\varepsilon$, $\pi+\varepsilon)$. We will conclude the proof showing that $\sin \theta Q_{\nu}^{u}(\theta, \varphi)$ must be a smooth function on $(\pi / 2-\varepsilon, \pi / 2+\varepsilon) \times(\pi-\varepsilon, \pi+\varepsilon)$. To this end choose an open neighborhood $U_{1}$ of a point of $(0, \pi) \times(0,2 \pi) \times T$ and an open neighborhood $U_{2}$ of the unit element of $S U(2)$ so that the map: $\left(\theta, \varphi, e^{i \psi}, u_{2}\right)$ $\rightarrow\left(\theta, \varphi, e^{i 2(\psi+t)}\right)$ defined by $\omega_{2}(\theta) \omega_{3}(\varphi) u_{2}=\omega_{3}(t) \omega_{3}\left(\theta^{\prime}\right) \omega_{3}\left(\varphi^{\prime}\right)$ is smooth on $U_{1} \times U_{2}$ and that for each $\left(\theta, \varphi, e^{i \psi}\right) \in U_{1}$ the map: $u_{2} \rightarrow\left(\theta^{\prime}, \varphi^{\prime}, e^{i(\psi+t)}\right)$ from $U_{2}$ into $(0, \pi) \times(0,2 \pi) \times T$ is a diffeomorphism. It turns out that the restriction $\omega_{i}^{\pi}, u \mid \mathfrak{S}_{0}^{\pi, u}$ is of the form

$$
\omega_{i}^{\pi, u}=\left(a_{i 1} \partial_{\theta}+a_{i 2} \partial_{\varphi}+a_{i 3} \partial_{\psi}\right),
$$

where $a_{i j}(i, j=1,2,3)$ are real-valued $C^{\infty}$-functions depending only on $(\theta, \varphi)$ with $\operatorname{det}\left(a_{i j}\right) \neq 0$. Now it is not difficult to see that $\sum_{i}\left(\hat{\omega}_{i}^{\pi}, u\right)^{2}$ is an elliptic differential operator with $C^{\infty}$-coefficient and that each $f_{\nu} Q_{\nu}^{u} e^{i \nu \psi}$ satisfies (17), from which the smoothness of $\sin \theta Q_{\nu}^{u}(\theta, \varphi)$ follows. Q.E.D.

We summarise the $k$-th heighest weight vectors $W_{k}$ for the representations $U^{\pi, e}$.

\begin{tabular}{|l|c|c|c|}
\hline$\pi$ & $\ell$ & $W_{k}(\neq\{0\})$ & $k$ \\
\hline$\pi_{(\ell, 0)}$ & $\ell=-1 / 2+i \rho, \rho \geqslant 0$ & $\sum_{\nu=-k}^{k} f_{\nu} P_{-\nu} e^{-i k \varphi+i \nu \psi}$ & $0,1, \cdots$ \\
\hline$\pi_{(\ell, 1 / 2)}$ & $\ell=-1 / 2+i \rho, \rho>0$ & $\sum_{\nu=-k}^{k} f_{\nu} P_{-\nu} e^{-i k \varphi+i(\nu+1 / 2) \psi}$ & $1 / 2,3 / 2, \ldots$ \\
\hline$\pi_{(\ell, 0)}$ & $-1<\ell<-1 / 2$ & $\sum_{\nu=-k}^{k} f_{\nu} P_{-\nu} e^{-i k \varphi+i \nu \varphi}$ & $0,1, \cdots$ \\
\hline$\pi_{(\ell, 0)}^{+}$ & $\ell=-1,-2, \cdots$ & $\sum_{\nu=-\ell}^{k} f_{\nu} P_{-\nu} e^{-i k \varphi+i \nu \psi}$ & $-\ell,-\ell+1, \cdots$ \\
\hline$\pi_{(\ell, 1 / 2)}^{+}$ & $\ell=-1 / 2,-3 / 2, \cdots$ & $\sum_{\nu=-\ell}^{k} f_{\nu} P_{-\nu} e^{-i k \varphi+i(\nu+1 / 2) \psi}$ & as above \\
\hline$\pi_{(\ell, 0)}^{-}$ & $\ell=-1,-2, \cdots$ & $\sum_{\nu=\ell}^{-k} f_{\nu} P_{-\nu} e^{-i k \varphi+i \nu \psi}$ & as above \\
\hline$\pi_{(\ell, 1 / 2)}^{-}$ & $\ell=-1 / 2,-3 / 2, \cdots$ & $\sum_{\nu=\ell}^{-k} f_{\nu} P_{-\nu} e^{-i k \varphi+i(\nu+1 / 2) \psi}$ & as above \\
\hline
\end{tabular}

(Here we put $P_{-\nu}=P_{k,-\nu}^{k}$ ) 
Denote $W_{k}^{0}$ a subspace of $W_{k}$ consisting of functions expressible as (14). Making use of formulas (chap. 3, sec 4 [17])

$$
\begin{gathered}
\partial_{\theta} p_{m, n}^{k}(\cos \theta)=\frac{i}{2}\left(\sqrt{(k+n+1)(k-n)} P_{m, n+1}^{k}(\cos \theta)\right. \\
\left.+\sqrt{(k+n)(k-n+1)} P_{m, n-1}^{k}(\cos \theta)\right), \\
i(m-n \cos \theta) P_{m, n}^{k}(\cos \theta)=\frac{\sin \theta}{2}\left(\sqrt{(k+n)(k-n+1)} P_{m, n-1}^{k}(\cos \theta)\right. \\
\left.-\sqrt{(k-n)(k+n+1)} P_{m, n+1}^{k}(\cos \theta)\right),
\end{gathered}
$$

and calculating formally, we see that

$$
\begin{aligned}
& \Delta^{\prime}\left(\sum_{\nu \geqslant-\ell}^{k} f_{\nu} P_{k,-\nu}^{k} e^{-i k \varphi+i \nu \psi}\right)=\sum_{\nu \geqslant-\ell}^{k}\left[-2 i \nu\left(\partial_{\tau}+\operatorname{th} \tau\right) f_{\nu}\right. \\
& -(\ell+\nu+1) \sqrt{(k+\nu+1)(k-\nu)} \frac{f_{\nu+1}}{\operatorname{ch} \tau} \\
& \left.\quad+(\ell-\nu+1) \sqrt{(k-\nu+1)(k+\nu)} \frac{f_{\nu-1}}{\operatorname{ch} \tau}\right] P_{k,-\nu}^{k} e^{-i k \varphi+i \nu \psi}
\end{aligned}
$$

Similarly, applying the formulas (18) (19) and

$$
\begin{gathered}
\sin \theta P_{k,-\nu}^{k}=-2 i \sqrt{\frac{(k-\nu+1)(k+\nu+1)}{(2 k+1)(2 k+2)}} P_{k+1,-\nu}^{k+1}, \\
\sin ^{2} \frac{\theta}{2} P_{k, \nu+1}^{k}=-\sqrt{\frac{(k+\nu)(k+\nu+1)}{(2 k+1)(2 k+2)}} P_{k+1,-\nu}^{k+1}, \\
\cos ^{2} \frac{\theta}{2} P_{k,-\nu-1}^{k}=\sqrt{\frac{(k-\nu)(k-\nu+1)}{(2 k+1)(2 k+2)}} P_{k+1,-\nu}^{k+1}
\end{gathered}
$$

we obtain

$$
\begin{aligned}
& F_{+}\left(\sum_{\nu \geqslant-\ell}^{k} f_{\nu} P_{k,-\nu}^{k} e^{-i k \varphi+i \nu \psi}\right) \\
& =\frac{1}{\sqrt{(2 k+1)(2 k+2)}} \sum_{\nu \geqslant-\ell}^{k+1}[2 i \sqrt{(k-\nu+1)(k+\nu+1)} \\
& \quad \times\left(\partial_{\tau}-k \operatorname{th} \tau\right) f_{\nu}+(\ell+\nu+1) \sqrt{(k-\nu)(k-\nu+1)} \frac{f_{\nu+1}}{\operatorname{ch} \tau} \\
& \left.+(\ell-\nu+1) \sqrt{(k+\nu)(k+\nu+1)} \frac{f_{\nu-1}}{\operatorname{ch} \tau}\right] \\
& \quad \times P_{k+1,-\nu}^{k+1} e^{-i(k+1) \varphi+i \nu \psi} .
\end{aligned}
$$

Since $f$ in $W_{k}^{0}$ is $C^{\infty}$-function on $V_{i M}$, the formal calculus can be justified. 
Set $c_{\nu}=\left\|e^{i \nu \psi}\right\|_{\pi}$. The isometry $J_{k}$ from $W_{k}$ onto $\sum_{\nu \geqslant-\ell}^{k} \oplus L^{2}(R)$ defined by

$$
\sum_{\nu \geqslant-\ell}^{k} f_{\nu} P_{k,-\nu}^{k} e^{-i k \varphi+i \nu \varphi} \rightarrow\left(\sqrt{\frac{2}{2 k+1}} c_{\nu} f_{\nu}(\tau) \operatorname{ch} \tau\right)
$$

transforms $\Delta^{\prime} \mid W_{k}^{0}$ to $\dot{L_{k}^{\pi}}$ :

$$
\dot{L_{k}^{\pi}}=-2 i(\nu) \partial_{\tau}+\frac{1}{\operatorname{ch} \tau} V,
$$

where $(\nu)=\left(\begin{array}{lllll}k & & & & \\ k-1 & & & & \\ & & \ddots & & \\ & & \nu & & \\ & & & \ddots & \\ & & & & -\ell\end{array}\right)$ and $V$ is an hermitian matrix whose $(\nu, \nu+1)$ component is equal to $-\sqrt{(-\ell+\nu)(\ell+\nu+1)(k+1+1)}$. Since the symmetric operator $\dot{L}_{k}^{\pi}$ is essentially selfadjoint with domain $\sum_{\nu \geqslant-\ell}^{k} C_{0}^{\infty}(\boldsymbol{R})$ [7], we denote $L_{k}^{\pi}$ its selfadjoint extension. Now the following proposition is selfexplanatory.

Proposition 2. For the representation $\pi=\pi_{(\ell, 0)}^{+}$the restriction $\Delta^{\prime \pi, e} \mid W_{k}$ is unitarily equivalent to $L_{k}^{\pi}$ provided $k=-\ell,-\ell+1, \cdots$.

Similarly we have

Proposition 3. For the representation $\pi=\pi_{(\ell, 0)}$ either with $\ell=-1 / 2$ $+i \rho(\rho \geqslant 0)$ or with $-1<\ell<-1 / 2$, the restriction $\Delta^{\pi, e} \mid W_{0}$ is unitarily equivalent to $L_{0}^{\pi}$ which is the selfadjoint extension of a symmetric operator $\dot{L_{0}^{\pi}}$ on $L^{2}(R)$ with domain $C_{0}^{\infty}(R)$ :

$$
\dot{L_{0}^{\pi}}=-\partial_{\tau}^{2}-\frac{\ell(\ell+1)}{\operatorname{ch}^{2} \tau} .
$$

For a Borel set $B$ of $\boldsymbol{R}$ and $\sigma$-finite measure $\sigma$ on $B$, let $\int_{B}^{\oplus} \lambda d \sigma$ denote the $\lambda$-multiplication operator in $L^{2}(B, \sigma)$.

Proposition 4. (i) For the representation $\pi=\pi_{(\ell, 0)}^{+} L_{k}^{\pi}$ is unitarily equivalent to $[k+\ell+1] \int_{R}^{\oplus} \lambda d \lambda$. (ii) For the representation $\pi=\pi_{(\ell, 0)}$ either 
$\ell=-1 / 2+i \rho(\rho \geqslant 0)$ or with $-1<\ell<-1 / 2, L_{0}^{\pi}$ is unitarily equivalent to [2] $\int_{R_{+}}^{\oplus} \lambda d \lambda$.

Proof. Applying the result of [7], we obtain (i). We note that $L_{0}^{\pi}$ is a Schrödinger operator with a so-called short range potential. So (ii) is a direct consequence of Agmon [1] and Kato [9].

Proposition 5. For the representation $\pi=\pi_{(\ell, 0)}^{+}, \Delta^{\prime \pi, e} \mid W_{k} \ominus F_{+}^{\pi, e} W_{k-1}$ is unitarily equivalent to $\int_{R}^{\oplus} \lambda d \lambda$ provided $k=-\ell,-\ell+1, \cdots$.

Proof. Lemma 4 and (i) of Proposition 4 yield the proposition.

Q.E.D.

For the representation $\pi=\pi_{(\ell, 0)}$ with $\ell=-1 / 2+i \rho(\rho \geqslant 0)$ or with $-1<\ell<-1 / 2 L_{k}^{\pi}$ is unitarily equivalent to [2k] $\int_{R}^{\oplus} \lambda d \lambda \oplus\left[\boldsymbol{\aleph}_{0}\right] \int_{\{0\}}^{\oplus} \lambda \delta(d \lambda)$ for any positive integer $k$, where $\delta$ denotes the Dirac measure. In order to show that $\Delta^{\prime \pi, e} \mid W_{k} \ominus F_{+}^{\pi, e} W_{k-1}$ is unitarily equivalent to [2] $\int_{R}^{\oplus} \lambda d \lambda$ we must check that $\Delta^{\prime \pi, e} \mid W_{k} \ominus F_{+}^{\pi, e} W_{k-1}$ has no eigenvectors with eigenvalue zero. This requires some calculation which we do not cite here. In this way we can manage to decompose the induced representations $\underset{S U(1,1) \uparrow S L(2, C)}{\text { Ind } \pi}$ (cf. [3] [13]).

\section{§5. Proof of Theorem 1 and 3}

We begin with

Lemma 10. Let $T_{t}$ and $S_{s}$ be one-parameter unitary groups on $L^{2}(R)$ :

$$
T_{t} f(\tau)=e^{i M t \operatorname{sh} \tau} f(\tau), \quad S_{s} f(\tau)=f(\tau+s) \quad(M \neq 0) .
$$

Then a closed subspace $D$ of $L^{2}(R)$ which is invariant with respect to $\left\{T_{t}: t \geqslant 0\right\}$ and $\left\{S_{s}: s \in R\right\}$ is either $L^{2}(R)$ or the null space $\{0\}$.

Proof. Denote $\hat{f}$ the Fourier transform of $f$. Since $D$ is $S_{s}$-invariant, there exists a Borel set $B$ such that $D=\left\{f \in L^{2}(R): \hat{f}(\lambda)=0\right.$ on the complement $\left.B^{c}\right\}$. If the Lebesgue measure $|B|$ is equal to zero, we have nothing to do. Otherwise, from the fact that Laplace transform $G_{\alpha}=$ $\int_{R_{+}} e^{-\alpha t} T_{t} d t$ is just the multiplication $1 /(\alpha-i M \operatorname{sh} \tau)$ it follows that for 
non-zero element $f$ of $D$ Fourier transform of $G_{\alpha} f \in D$ is a non-zero holomorphic function on the strip $|\operatorname{Im} \lambda|<1$. Thus $\left|B^{c}\right|=0$. Q.E.D.

Proof of Theorem 1. First note that Theorem 2 also holds for the 2dimensional space-time Poincaré group. Irreducible unitary representations corresponding to space-like orbits $V^{ \pm i M}(2)=\left\{\hat{x}_{0}^{2}-\hat{x}_{3}^{2}=-M^{2}: \hat{x}_{3} \gtrless 0\right\}$ have the realization in $L^{2}(R)$ :

$$
U^{i M}\left(\left(x_{0}, x_{3}\right), \omega_{6}(s)\right) f(\tau)=\exp \left( \pm i M\left(x_{0} \operatorname{sh} \tau+x_{3} \operatorname{ch} \tau\right)\right) f(\tau+s) .
$$

Now Lemma 10 yields the theorem.

Q.E.D.

Let us turn to the proof of Theorem 3. As in $\S 4, W_{k}$ stands for the $k$-th heighest weight vectors corresponding to the representation $\left(U^{\pi, e} \mid G, \mathfrak{S}_{2}^{\pi}\right)$ of $G=S L(2, C)$. Denote $k_{0}$ the minimum of $\left\{k: W_{k} \neq\{0\}\right\}$. We observe

Lemma 11. If there exists an invariant non-trivial closed subspace $D_{+}$ of $\mathfrak{S}^{\pi}$ with respect to the Poincaré subsemigroup $P_{+}$, then there exists a non-trivial closed subspace $D$ of $W_{k_{0}}$ which is invariant with respect to $\left\{\boldsymbol{T}_{t}=e^{i M t \operatorname{sh} \tau}: t>0\right\}$ and $\left\{e^{i t s}, e^{i s s^{\prime}}: s \in \boldsymbol{R}\right\}$.

Proof. Our reasoning depends on the results of $\S 3$. Denoting the orthogonal complement of $D_{+}$by $D_{+}^{\perp}$, it holds that

$$
W_{k_{0}}=\left(W_{k_{0}} \cap D_{+}\right) \oplus\left(W_{k_{0}} \cap D_{+}^{\perp}\right) \text {. }
$$

We know that $W_{k_{0}} \cap D_{+}$(resp. $\left.D_{+}^{\perp}\right)$ is invariant with respect to $T_{t}(t>0)$ resp. $t<0), \Delta$ and $\Delta^{\prime}$. Thus both components on the right side of (25) have the same property. We claim none of them is a null space. We will show this for $W_{k_{0}} \cap D_{+}$. The proof for the another component is similar. If $W_{k_{0}} \cap D_{+}$is a null space, some $k, k \geqslant k_{0}$ attains the maximum of $\left\{k^{\prime}: W_{k^{\prime}} \cap D_{+}=\{0\}\right\}$. Since the decomposition (25) holds for any $k, W_{k}$ is a subspace of $D_{+}^{\perp}$. Thus $F_{+} W_{k}^{0}$ and $F_{+} \bar{G}_{\alpha} W_{k}^{0}$ are orthogonal to $W_{k+1} \cap$ $D_{+}$, where $\bar{G}_{\alpha}$ denotes Laplace transform $\int_{R_{+}} e^{-\alpha t} T_{-t} d t=1 /(\alpha+i M \operatorname{sh} \tau)$. An $f \in J_{k+1}\left(W_{k+1} \cap D_{+}\right)$satisfies

$$
\left(f, J_{k+1} F_{+} J_{k}^{-1} h\right)=0, \quad\left(G_{\alpha} f, J_{k+1} F_{+} J_{k}^{-1} h\right)=0 \quad \text { for any } h \in J_{k} W_{k}^{0}
$$

(see (22) for $J_{k}$ ). From the second equality it follows that

$$
\left(A \frac{i M \operatorname{ch} \tau}{(\alpha-i M \operatorname{sh} \tau)^{2}} f, \check{h}\right)+\left(f, J_{k+1} F_{+} J_{k}^{-1} \bar{G}_{\alpha} h\right)=0 \quad \text { for any } h \in J_{k} W_{k}^{0},
$$

where $A$ is a constant diagonal matrix whose $(\nu, \nu)$ component is equal to $2 i \sqrt{(k-\nu+1)(k+\nu+1)} / \sqrt{(2 k+2)(2 k+3)}$ and $\breve{h}$ denotes $\left(0, h^{t}\right)^{t} \in J_{k+1} W_{k}^{0}$. 
Since the second term of (27) vanishes, $f_{\nu}$ is zero except $f_{k+1}$. Together with the first equality of (26) $f$ vanishes. This completes the proof.

Q.E.D.

Proof of Theorem 3. For the representation $U^{\pi, e}$ (see (6)) with, say $\pi=\pi_{(\ell, 0)}^{+}, W_{k_{0}}$ coincides with $W_{-\ell}$. Since $J_{k_{0}}$ transforms $T_{t}$ and $\Delta^{\prime}$ to $T_{t}$ and $2 i \ell \partial_{\tau}$ respectively, the theorem follows from Lemma 10 and 11 .

Q.E.D.

Acknowledgement. The author expresses his sincere thanks to Professors Takenaka and Tatsuuma for their kind advice and to Mr. Itatsu for his interest in the problem.

\section{REFERENCES}

[1] S. Agmon, Spectral properties of Schrödinger operators and scatttering theory, Annali dela Scuola Normal Superiore di Pisa, series 3, 2 (1975), 149-218.

[2] E. Angelopoulos, Decomposition sur le sou-groupe de Poincaré de la represéntation de mass positive et de spin nul du groupe de Poincaré. Ann. Inst. Henri Poincaré XV, no. 4 (1971), 303-320.

[ 3 ] - Reduction on the Lorentz subgroup of UIR's of the Poincaré group induced by a semisimple little group, Math. Phys. 15 (1974), 155-165.

[ 4 ] J. Dixmier, Les $C^{*}$-algèbres et leurs represéntations. Gauthier-Villars Paris, 1969.

[5] A. Erdélyi, Higher transcendental functions 1, McGraw-Hill, 1955.

[6 ] L. Hormander, Linear partial differential operators, Springer, 1963.

[7] S. Itatsu and H. Kaneta, Spectral properties of first order ordinary differential operators, to appear.

[ 8 ] H. Joos, Zur Darstellungstheorie per inhomogeneous Lorentzgruppe als Grundlage quantenmechanischer Kinematik, Fortschr. Phts. 10 (1962), 65-146.

[9] T. Kato, Growth properties of solutions of reduced wave equation with a variable coefficient, Comm. Pure Appl. Math. 12 (1959), 403-425.

[10] G. W. Mackey, Induced representations of locally compact group I, Ann. of Math. 55, no. 1 (1951), 101-139.

[11] P. D. Lax and R. S. Phillips, Scattering theory, Academic Press, 1967.

[12] M. A. Naimark, Linear representation of Lorentz group, VEB Deucher Verlag der Wissenschaften Berlin, 1963.

[13] B. Radhakrishnan and N. Mukunda, Spacelike representations of the inhomogeneous Lorentz group in a Lorentz basis, J. Math. Phys. 15 (1974), 477-490.

[14] I. E. Segal, A class of operator algebras which are determined by groups, Duke Math. J. 18 (1951), 221-265.

[15] N. Tatsuuma, Decomposition of representations of three-dimensional Lorentz group, Proc. Japan Acad. 38 (1962), 12-14.

[16] —, Decomposition of Kronecker products of representations of inhomogeneous Lorentz group, Proc. Japan Acad. 38 (1962), 156-160.

[17] N. Vilenkin, Special functions and the theory of group representations, AMS translation of monographs 22, 1968.

Department of Mathematics

Nagoya University 